\title{
Editorial
}

\section{Diabetologia and the Diabetologists}

Diabetologia prides itself in being an interdisciplinary journal which publishes reports in all areas pertinent to the cure, care and prevention of diabetes in its different forms. This contention includes applied (clinical) as well as basic science. The driving force for such broad coverage in a single journal is both today's increasing prevalence of diabetes, which at present is estimated to be $3 \%$ worldwide and to affect 150 million of the 6 billion people on earth, and our inability to contain the disease. The World Health Organisation predicts that the number of patients suffering from diabetes will double by the year 2025 .

Without a doubt the increasing prevalence of the disease over the last 50 years is predominantly caused by environmental factors. In the case of Type 2 diabetes these are not infectious agents of some sort but changes in lifestyle such as over-nutrition and not enough energy expenditure due to only limited physical exercise. The worldwide spread of a westernised lifestyle promotes obesity-driven diabetes for all age categories, affecting the elderly and increasingly children as well.

We are therefore definitely confronted by a diabetes epidemic on the rise. It puts diabetologists in high demand for the years to come. But what are the characteristics of a diabetologist? In an attempt to answer this question and to describe diabetologists in more detail I turned to the table of contents of Diabetologia, a journal which strives to cover all fields of expertise pertinent to diabetes. I also checked the titles of articles published in the preceding year. To my surprise I came up with a list of expertise which goes far beyond the listed subheadings of Diabetologia and which covers a remarkable area of both basic and clinical science (Table 1).

Published online: 14 January 2003

(C) Springer-Verlag 2003
I was overwhelmed by the clinical and scientific demands put on a diabetologist and identified three subgroups: the physician diabetologist, the clinical diabetology scientist and the basic scientist interested in diabetes.

The physician diabetologist in charge of patient care has to command clinical skills going far beyond the still too common attitude of glucose-centrism, which just focuses on treating elevated blood glucose concentrations and abnormal $\mathrm{HbA}_{1 \mathrm{c}}$. To serve his patients well, the modern physician diabetologist has to be more of an internist than just a blood glucosecentred diabetologist. He has to care not only for good metabolic control but also for maintenance of normal blood pressure, kidney function, vascular patency, endothelial and gastrointestinal function, and among others sexual health. In addition, he has to help prevent the progression of the disease in the patient's diabetic heart and diabetic foot. Against this background clinical diabetology has to be recognised as a demanding job whereby the physician is required to be knowledgeable in a considerable number of sub-specialities of Internal Medicine. He has to be thoroughly trained to be able to serve the patient properly and to co-ordinate any necessary support by specialists of all sorts. The dimension of this task has not been fully perceived by many clinical practitioners involved in diabetes care. Diabetologia tries to help the situation by providing its series of reviews.

The diabetologist scientist is confronted with similarly formidable expectations. He has to be knowledgeable both in Internal Medicine or Paediatrics and in Clinical Pharmacology, which includes study design and complex methodologies borrowing from many sub-specialities depending on the hypotheses tested. This can become a difficult task when the diabetologist scientist turns to studies of more complex cardiological, neurological or ophthalmological problems as they 
Table 1. Diabetology required expertise

Expertise (incomplete) as defined by Diabetologia's content

\begin{tabular}{lll}
\hline Defined by subheadings & Basic Science & Clinical Science \\
\hline Epidemiology & & \\
Clinical trials and diabetes care & Animal Experimentation & Angiology \\
Aetiology and pathophysiology & Biochemistry & Cardiology \\
Metabolism & Blood clotting & Clinical pharmacology \\
Immunology & Cytology & Dermatology \\
Islets of Langerhans & Electrophysiology & Economics of health care \\
Diabetes associated complications & Embryology & Endocrinology and Metabolism \\
Genetics & Molecular biology & Gastroenterology \\
Methodology & Oxidative stress & Infectiology \\
& Pharmacology & Nephrology \\
& Physiological chemistry & Paediatrics \\
& Stable isotope technique & Preventive Medicine
\end{tabular}

occur in the course of diabetes. Clearly, the diabetologist has to be a good physician but also an excellent clinical investigator. Such experts are in high demand in clinical science, but they are few in number and for many years have been regarded as an endangered species [1]. They require utmost protection and support in any clinical and academic environment. Their training should be a major obligation for units of clinical diabetology, which could become a major provider of highly qualified clinical investigators.

The third group of diabetologists is the basic scientist interested in fundamental diabetes research. $\mathrm{He}$ can be found in a multitude of basic science areas (Table 1). Such experts are not burdened and limited in their efforts by clinical constraints. They study metabolic and other derangements as they occur in a diabetic organism in molecular detail and they have their own jargon, which occasionally can be hard to follow for the clinician. Their invaluable work, however, brings understanding and insights in detail and contributes about $50 \%$ of published articles to Diabetologia.

Having said this, the question comes up as to why basic and clinical scientists should agree to have their work published back to back in the same journal? The key words to the answer are 'dialogue' and 'cross fertilisation' between different areas of expertise. These require interpretation of knowledge and thinking in analogies, which to some extent is a clinical art. Such interaction can provide an in depth understanding of clinical findings and can help formulate questions for more detailed analysis of the basic mechanisms underlying the heterogenous syndrome of diabetes mellitus.

Indeed, diabetes refers to a group of complex diseases whose study requires institutions to provide close interaction of clinical and basic sciences, as well as expert training to diabetologists of all three groups. However, not only diabetologists need to be highly qualified, but the readers of Diabetologia are also confronted with the same demand. They are expected to have a keen interest in diabetes as well as in a broad spectrum of scientific disciplines.

To attract such readers, reports on diabetes research in a multidisciplinary journal like Diabetologia should provide excellent data. In addition, a beautifully and clearly written Abstract or last paragraph in the Discussion section can help seduce the reader to read the full text of an article. A basic requirement to ensure the reader's intellectual fun is also an understandable, well-defined and well-argued hypothesis. Reports should be made accessible by an adequate Introduction, which positions the proposed hypothesis properly in the spectrum of unsolved problems in the respective field of diabetes research and care. An acceptance rate of only $20 \%$ helps Diabetologia to meet these demands and to attract the attention of our readers. I thank the authors for submitting reports to Diabetologia which have met its stringent criteria for acceptance and which have thereby contributed to the improving quality of the jurnal.

To better meet the needs of patients suffering from diabetes and to entice the readers of Diabetologia so that physician diabetologists, clinical investigators and basic scientists alike eagerly await the journal's next monthly issue and fully read it, the influx of high quality manuscripts should, however, be increased even further.

To achieve this goal I encourage all investigators active in diabetes research to continue submitting their best work to Diabetologia.

Werner K. Waldhäusl

Editor-in-Chief

\section{References}

1. Wyngaarden JB (1979) The clinical investigator as an endangered species. N Engl J Med 301:1254-1259 